\title{
Interacciones medicamentosas en pacientes bajo tratamiento con itraconazol para diferentes tipos de micosis
}

\author{
Adriana L. Fernández¹, Diana L. Hernández¹, Angela M. Londoño ${ }^{1}$, Carolina López ${ }^{1}$, Yesid Pineda \\ Juan D. Wolff' ${ }^{1}$, María I. Múnera², Elsa M. Zapata ${ }^{3}$
}

\begin{abstract}
Resumen
Algunos estudios han reportado un aumento en la frecuencia e importancia de las interacciones del itraconazol en pacientes bajo polifarmacia con fármacos que afectan el sistema enzimático de la citocromo P450 y modifican el metabolismo del azol. Con el propósito de determinar las características epidemiológicas, farmacológicas y clínicas de las interacciones medicamentosas en pacientes bajo tratamiento con itraconazol para diferentes tipos de micosis, se realizó un estudio descriptivo transversal retrospectivo en el que se analizaron 21 historias clínicas de pacientes atendidos en la Corporación para Investigaciones Biológicas durante el período 1994-1997. Entre la población estudiada, seis pacientes presentaron paracoccidioidomicosis; cinco, cromoblastomicosis; cuatro, histoplasmosis; cuatro, criptococosis, y dos, aspergilosis. En promedio, la dosis de itraconazol, la duración y el número de fármacos recibidos concomitantes fueron 223,8 mg/día, 44,3 semanas y 2,7 fármacos, respectivamente. En 10 individuos, se presentaron enfermedades de base y en 15, enfermedades concomitantes. Se documentó interacción medicamentosa (niveles no detectables o no terapéuticos de itraconazol) en 18 pacientes, de los cuales 17 presentaban sospecha clínica y 14 sospecha paraclínica de interacción. El medicamento más frecuentemente involucrado con interacción fue la rifampicina, seguido de los inhibidores $\mathrm{H}_{2}$, antiácidos y fenitoína. Similar a lo encontrado en otros estudios, la administración concomitante de estos fármacos con el itraconazol, reduce los niveles séricos de este último y altera la respuesta clínica al tratamiento. Este estudio es una aproximación inicial, aún no realizada en nuestra población, que permitirá establecer protocolos para el manejo y prevención de interacciones medicamentosas en pacientes en tratamiento con itraconazol y otros fármacos.
\end{abstract}

Palabras clave: itraconazol, interacción medicamentosa.

Drug interactions of itraconazole in patients under treatment for different mycoses

\section{Abstract}

Some studies have reported an increasing number of clinically significant interactions of itraconazole in patients receiving drugs known to have profound effects on the cytochrome P-450 enzyme system. With the purpose of determining the epidemiological, pharmacological and clinical characteristics of drug interactions in patients under therapy

\footnotetext{
${ }^{1}$ Facultad de Medicina, Pontificia Universidad Bolivariana, Medellín, Colombia.

2 Clínica Cardiovascular, Medellín, Colombia.

${ }^{3}$ Corporación para Investigaciones Biológicas, Medellín, Colombia.
} 
with itraconazole, twenty-one patients with clinical and laboratory information suspicious of interaction were included in this transversal retrospective study undertaken during the period of 1994-1997. Six patients presented paracoccidioidomycosis, five chromoblastomycosis, four histoplasmosis, four cryptococcosis and two aspergillosis. Most of patients had either an underlying or concurrent illness like AIDS or tuberculosis. The average dose of itraconazole was $223.8 \mathrm{mg} /$ day, the mean duration of therapy was 23.1 weeks and each patients received around 2.6 drugs simultaneously administered with itraconazole. Drug interaction (undetectable or non-therapeutic serum concentrations of itraconazole) was found in 18 patients and rifampin, $\mathrm{H}_{2}$ blockers, antiacids and phenytoin were the most common drugs implicated. According to some published articles, the concurrent administration of these drugs and itraconazole disturbed the absorption and metabolism of this antifungal, subsequently decreased its serum concentration and provoked therapeutic failure. This study is an initial approach that will allow to establish clues for the management of patient under therapy with itraconazole and other therapies and at risk of developing drug interactions.

Key words: itraconazole, drug interaction.

\section{Introducción}

Los pacientes críticamente enfermos, como aquellos con virus de inmunodeficiencia humana (VIH), falla renal, hepática o respiratoria y diabetes mellitus, entre otros, requieren polifarmacia, (dos o más medicamentos) y, por tanto, tienen mayor riesgo de experimentar interacciones medicamentosas. La incidencia general de interacciones varía de 7,6 a $51,7 \%$ y la probabilidad de ocurrencia del fenómeno es directamente proporcional al número de medicamentos que recibe el individuo; así, en pacientes que reciben hasta 5 medicamentos, es de $4 \%$, en quienes reciben 6 a 10 fármacos Ilega a $10 \%$ y cuando se administran más de 16 medicamentos simultáneamente alcanza a $54 \%$ (1). Las interacciones medicamentosas se pueden manifestar clínicamente como aumento en la frecuencia y severidad de los efectos tóxicos de los medicamentos o falla terapéutica por disminución en su concentración sérica, lo que en algunos casos compromete la vida del paciente.

La alta prevalencia de infecciones micóticas en pacientes con defectos en sus mecanismos de defensa, ha revelado la necesidad de una terapia antifúngica segura y efectiva. El itraconazol, un fármaco triazólico oralmente activo, ha demostrado un amplio espectro de actividad y un favorable perfil farmacocinético. Está indicado en el tratamiento de una amplia gama de micosis superficiales y profundas como histoplasmosis, paracoccidioidomicosis, cromoblastomicosis, aspergilosis y criptococosis, entre otras (2).

En algunos estudios se ha reportado aumento en la frecuencia e importancia de las interacciones del itraconazol en pacientes bajo polifarmacia. Tucker y colaboradores en un estudio multicéntrico en Texas y California, describieron interacciones medicamentosas representadas en la disminución de las concentraciones séricas terapéuticas de itraconazol, ketoconazol o fluconazol que eran administrados para el tratamiento de micosis sistémicas en 12 pacientes con condiciones de base o concomitantes (trasplantes, diabetes mellitus, SIDA, falla renal y coinfección por micobacterias) y quienes, a su vez, recibían rifampicina, carbamazepina o fenitoína (3).

El principal mecanismo de interacción del itraconazol es el de tipo farmacocinético. Medicamentos como los antagonistas de receptores $\mathrm{H}_{2}$, antiácidos, inhibidores de la bomba de protones y didanosina, aumentan el $\mathrm{pH}$ gástrico y limitan la absorción del itraconazol. A su vez, los medicamentos que inducen la familia CYP3A4 de las enzimas del sistema citocromo P450, entre ellos la rifampicina y la fenitoína, aceleran su metabolismo y disminuyen sus concentraciones séricas a niveles no terapéuticos 
según el tipo de micosis $(2 \mu \mathrm{g} / \mathrm{mL}$ para histoplasmosis, $5 \mu \mathrm{g} / \mathrm{mL}$ para aspergilosis y $1 \mu \mathrm{g} / \mathrm{mL}$ para criptococosis, determinados por método de bioensayo).

En la Corporación para Investigaciones Biológicas (CIB) se viene evaluando, desde 1994, el itraconazol en el tratamiento de diversas micosis $y$ aunque se han detectado interacciones medicamentosas en algunos pacientes, no se ha establecido su frecuencia, tipos, tiempo de aparición, ni consecuencias. Este estudio descriptivo transversal retrospectivo, se propuso hacer una aproximación inicial, realizada por primera vez en nuestra población, sobre las características epidemiológicas, farmacológicas y clínicas de las interacciones medicamentosas en un grupo de 21 pacientes bajo tratamiento con itraconazol y otros fármacos, quienes fueron remitidos a la $\mathrm{CIB}$ en el período comprendido entre enero de 1994 y diciembre de 1997.

\section{Materiales y métodos}

\section{Tipo de estudio}

Se realizó un estudio de tipo descriptivo transversal retrospectivo en una población de pacientes remitidos a la CIB de enero de 1994 hasta diciembre de 1997. Estos pacientes recibieron tratamiento con itraconazol para diferentes tipos de micosis y presentaron sospecha clínica o paraclínica de interacción con otros medicamentos recibidos concomitantemente.

\section{Pacientes}

La población estuvo conformada por 84 pacientes y la muestra no probabilística consecutiva de 21 de éstos, tras cumplir con los siguientes criterios de inclusión:

- diagnóstico de micosis bajo tratamiento con itraconazol;

- niveles séricos de itraconazol medidos por método de bioensayo;

- terapia combinada con otros fármacos;

- datos completos consignados en la historia clínica que permitieran hacer el análisis;

- presencia de interacción medicamentosa.
Esta se definió como la demostración de niveles séricos de itraconazol inferiores a los recomendados o no terapéuticos, circunstancia que impide el control efectivo de la micosis bajo tratamiento. Es de anotar que existen variaciones de acuerdo con el tipo de micosis; así, por el método del bioensayo, el rango para la histoplasmosis es de $2 \mu \mathrm{g} / \mathrm{mL}$, para la aspergilosis de $5 \mu \mathrm{g} / \mathrm{mL}$ y para la criptococosis, de $1 \mu \mathrm{g} / \mathrm{mL}$. Los valores para cromoblastomicosis y paracoccidioidomicosis se asumieron según los patrones presumidos de susceptibilidad al itraconazol.

- enfermedades de base: se consideraron como enfermedades de base aquéllas que predispusieron al inicio de la micosis tales como $\mathrm{VIH}$-SIDA y diabetes mellitus, entre otras.

- enfermedades concomitantes: se definieron como aquellas enfermedades que coexistían con la micosis sin predisponer a su aparición, pero que determinaron el tipo de fármacos que recibió el paciente o que podían alterar la farmacocinética del itraconazol, bien sea en su absorción, metabolismo, distribución o excreción.

- síntomas y signos clínicos sospechosos de interacción, según el médico tratante: se consideró la ausencia de mejoría o el empeoramiento clínico del paciente.

- signos paraclínicos sospechosos de interacción: se tomaron como tales la persistencia del examen directo, cultivo y serología positivas o la no negativización de los mismos.

\section{Métodos}

Las historias clínicas sirvieron de fuente secundaria para el formulario diseñado para tal efecto. La cuantificación sérica de itraconazol se realizó por el método de bioensayo referenciado por J.S.Hostetler (4) y estandarizado en el Laboratorio de Farmacología de la CIB. Las pruebas de laboratorio se realizaron con las técnicas estandarizadas en la CIB, para todas las micosis, examen directo y cultivo, y las pruebas serológicas que incluyeron la inmunodifusión y la fijación del complemento para histoplasmosis, paracocci- 
dioidomicosis y aspergilosis, y aglutinación en látex para el antígeno capsular en la criptococosis (5-8).

\section{Resultados}

De un total de 84 historias de pacientes tratados con itraconazol, se estudiaron 21 pacientes que cumplieron los requisitos de inclusión; 19 fueron hombres $(90,4 \%)$ y 2 mujeres $(9,5 \%)$, con una edad promedio de 45,04 $\pm 16,5$ años. Trece pacientes $(61,9 \%)$ procedían del área urbana y 8 pacientes $(38,1 \%)$ de área rural (cuadro 1$)$.

Durante el período en el que se desarrolló este estudio, fueron tratados con itraconazol para diferentes tipos de micosis 84 pacientes, de los cuales 18 , es decir, $21,4 \%$, desarrollaron interacción definida por niveles de itraconazol no detectables o no terapéuticos según la micosis tratada (figura 1).

De los 21 pacientes que presentaron sospecha clínica o paraclínica de interacción medicamentosa, en 18 se confirmó su presencia $(85,7 \%)$. La ausencia de mejoría clínica, la persistencia de serología positiva y el empeoramiento clínico fueron los criterios sugestivos de interacción más comúnmente encontrados por el médico tratante.

Las micosis más frecuentemente encontradas fueron la paracoccidioidomicosis y la cromoblastomicosis. La dosis promedio de itraconazol administrada a los pacientes diariamente fue $223,8 \mathrm{mg} /$ día, $25 \%$ y $75 \%$ de la población, correspondientes a $Q_{1}$ y a $Q_{3}$ recibieron $200 \mathrm{mg} /$ día y el promedio de semanas de tratamiento fue de 44,3 semanas, $25 \%\left(Q_{1}\right), 7$ semanas y $75 \%\left(Q_{3}\right), 42$ semanas. Con respecto a los fármacos que fueron administrados concomitantemente, en promedio cada paciente recibió 2,7 fármacos; $25 \%\left(Q_{1}\right), 1$ fármaco y $75 \%\left(Q_{3}\right)$, 4 fármacos.

En once de los pacientes no se detectaron enfermedades de base $(54,5 \%)$, siete tenían VIHSIDA (31,8\%); uno diabetes mellitus $(4,5 \%)$, y dos, otros estados de inmunosupresión $(9,09 \%)$. Las micosis más frecuentemente encontradas en los pacientes con SIDA fueron la criptococosis meníngea (cuatro pacientes), la histoplasmosis diseminada (tres pacientes) e histoplasmosis pulmonar (un paciente); todos estos pacientes desarrollaron interacción medicamentosa y/o falla terapéutica. Los únicos casos en los cuales la micosis no estuvo asociada con una enfermedad de base fueron la paracoccidioidomicosis y la cromoblastomicosis.

Las enfermedades concomitantes más comunes fueron las respiratorias (tuberculosis) y gastrointestinales (enfermedad acidopéptica) con 27,5 y $24,1 \%$, respectivamente. Siete pacientes que recibieron rifampicina como parte del tratamiento antituberculoso, se les documentó interacción medicamentosa, asociada con criterios de sospecha clínica o paraclínica y, de los cinco pacientes con enfermedad acidopéptica, únicamente tres recibían tratamiento con inhibidores $\mathrm{H}_{2}$ (ranitidina) y antiácido (hidróxido de aluminio más hidróxido de magnesio). Estos últimos presentaron persistencia de cultivos positivos y de serología reactiva, ausencia de mejoría clínica o empeoramiento de la enfermedad.

\section{Discusión}

Este estudio encontró una incidencia de interacción medicamentosa del 21,4\% (18 pacientes), definida por niveles de itraconazol no detectables o no terapéuticos según la micosis tratada (figura 1). Sin embargo, teniendo en cuenta que la mayoría de los pacientes $(95,3 \%)$ recibieron cinco o menos fármacos, se esperaba una cifra menor de $4 \%$ de incidencia de interacción (figura 2 y cuadro 2). Lo anterior sugiere que para la aparición de interacción en este estudio, el número no es tan significativo como el tipo de medicamentos recibidos concomitantemente, otras circunstancias clínicas asociadas $y$, especialmente, el alto potencial de interacción del itraconazol (1,3,9-11).

De los 21 pacientes que presentaron sospecha clínica o paraclínica, en 18 se confirmó la presencia de interacción $(85,7 \%)$. La ausencia de mejoría clínica, la persistencia de serología positiva y el empeoramiento clínico fueron los criterios sugestivos de interacción más comúnmente encontrados por el médico tratante. De esto se deduce que la sospecha clínica predijo en la mayoría de los casos las alteraciones en los niveles séricos del itraconazol. 


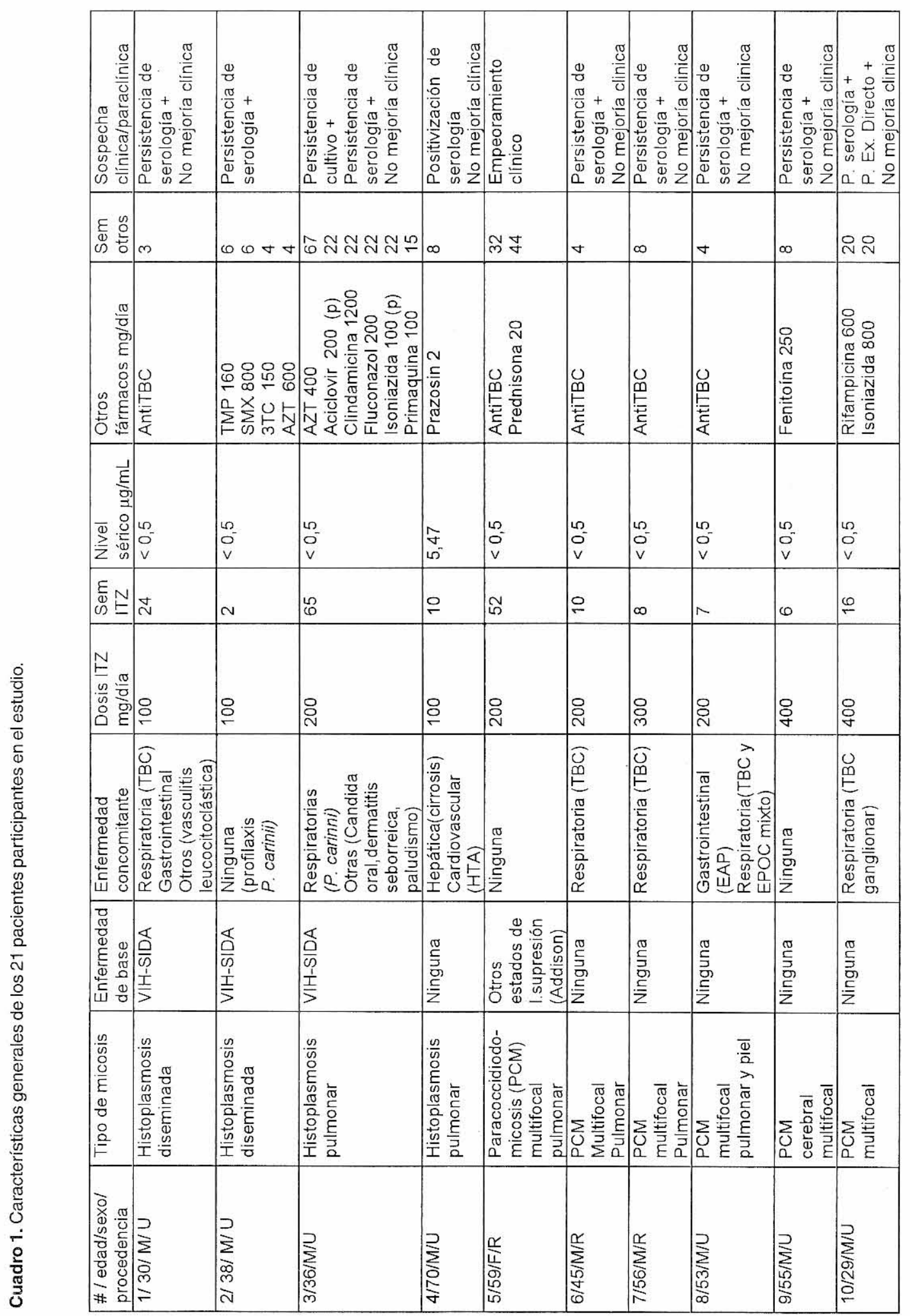




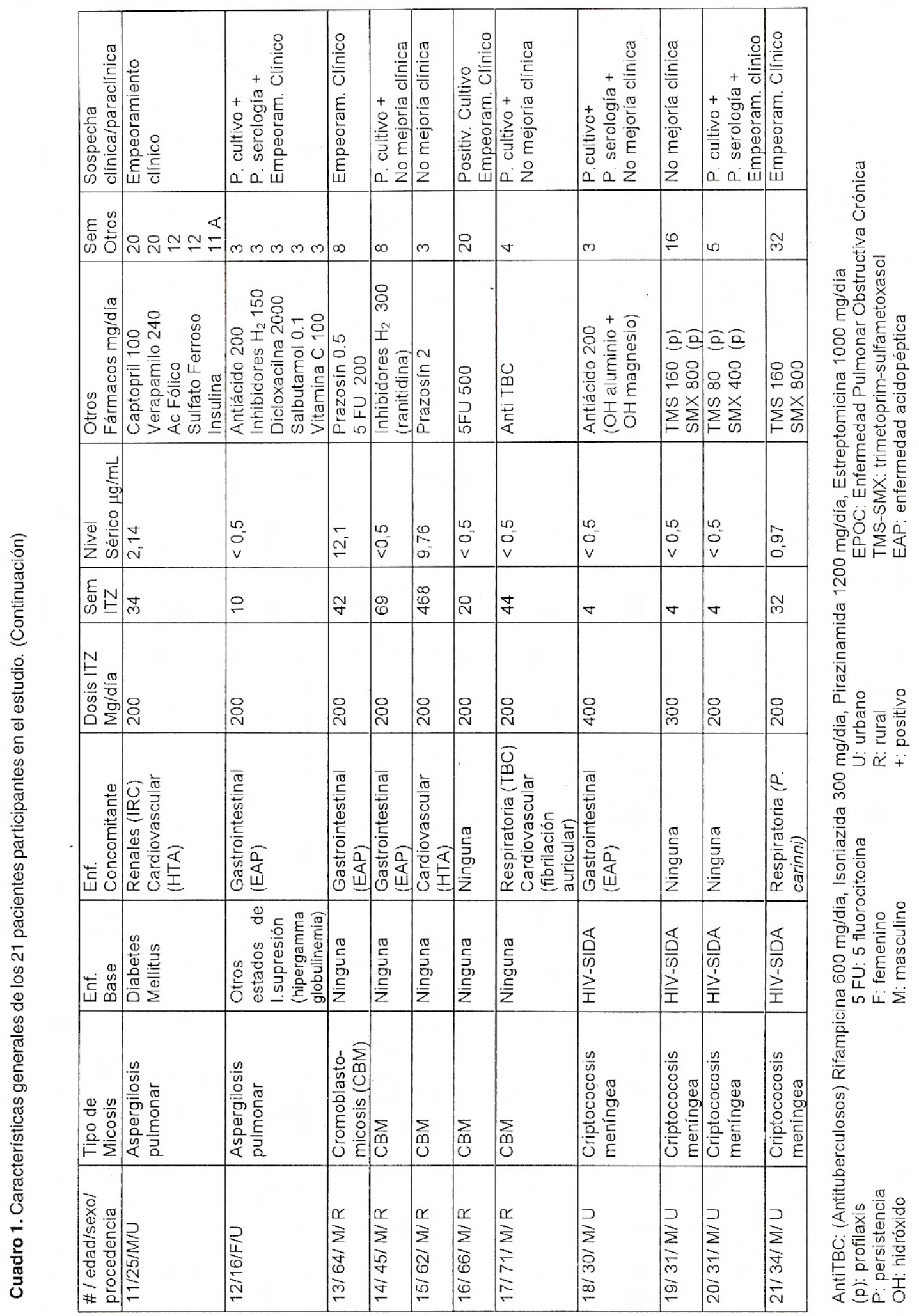




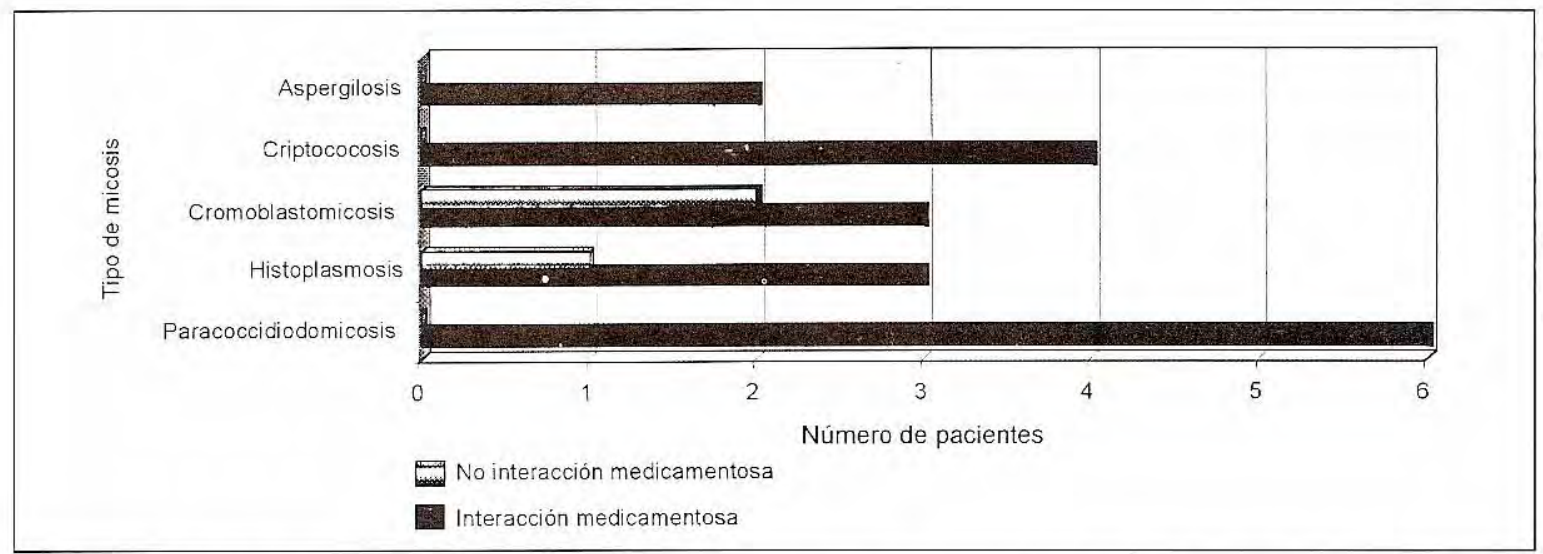

Figura 1. Distribución del número de pacientes con interacción medicamentosa según niveles séricos de itraconazol para cada tipo de micosis.

Niveles séricos de itraconazol $<5 \mu \mathrm{g} / \mathrm{mL}$ para aspergilosis y cromoblastomicosis, $<2 \mu / \mathrm{mL}$ para histoplasmosis y paracoccidioidomicosis $y<1 \mu \mathrm{g} / \mathrm{mL}$ para criptococosis, fueron tomados como interacción medicamentosa o falla terapéutica.

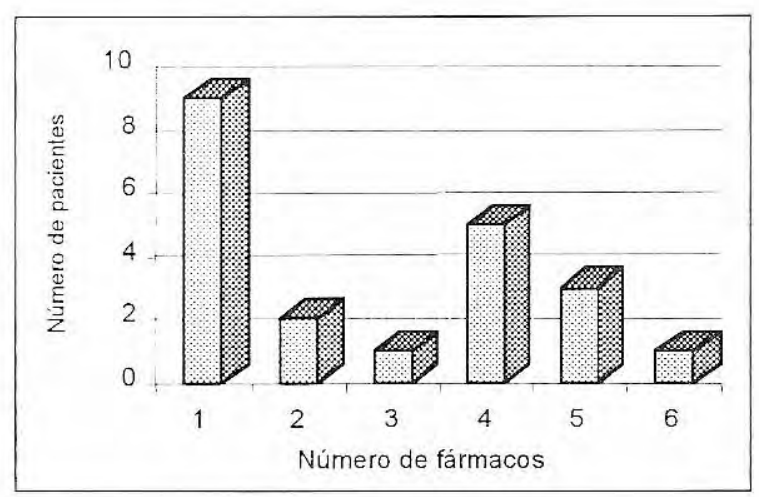

Figura 2. Distribución porcentual según número de fármacos administrados concomitantemente.

Con respecto a los fármacos que fueron administrados concomitantemente, en promedio cada paciente recibió 2,7 fármacos. El 42,9\% de los pacientes recibieron un fármaco, el $38,1 \%$ entre 2 y 4 fármacos y solo el $19 \%$ recibieron mas de cinco medicamentos concomitantemente al itraconazol.

Las micosis más frecuentemente encontradas en los pacientes con SIDA fueron la critopcocosis meníngea y la histoplasmosis diseminada y pulmonar, que se explica porque los defectos en los mecanismos de defensa mediados por células $T$ parecen tener un riesgo elevado de sufrir estas micosis $(5,7)$ y la pandemia de SIDA ha generado una verdadera explosión de éstas.

Las interacciones medicamentosas producidas por alteraciones del tracto gastrointestinal (hipoclorhidria y disminución de la superficie de absorción), administración de fármacos que aumentan el $\mathrm{pH}$ gástrico (antiácidos e inhibidores $\mathrm{H}_{2}$ ), alimentación nasogástrica, polifarmacia y mal estado general en pacientes con SIDA han sido descritas $(9,12,13)$. A su vez, la administración de inductores enzimáticos de la citocromo P450 CYP3A4, como la rifampicina, aceleran el metabolismo del itraconazol y subsecuentemente, disminuyen su concentración sérica $(3,14)$. La aparición de interacción en los siete pacientes con SIDA, puede explicarse por cualquiera de las situaciones señaladas anteriormente.

Los únicos casos en los cuales la micosis no estuvo asociada con una enfermedad de base fueron la paracoccidioidomicosis y la cromoblastomicosis, entidades que se caracterizan por una evolución bastante crónica y en las que no se describe la necesidad de algún factor o enfermedad de base para una mayor frecuencia en su presentación $(6,15)$. Situación contraria ocurre con la aspergilosis, cuyo determinante más importante es el estado inmune del paciente, no la intensidad de la exposición. Por otro lado, condiciones de base como la diabetes mellitus y la hipergammaglobulinemia, presente en ambos pacientes con aspergilosis, pudieron favorecer su aparición (8).

Las enfermedades concomitantes más comunes fueron la tuberculosis y la enfermedad acidopéptica. La asociación de tuberculosis con 
Cuadro 2. Distribución porcentual según tipo de fármaco administrado concomitantemente.

\begin{tabular}{|c|c|c|c|}
\hline \multicolumn{2}{|c|}{ Tipo de farmaco } & \multirow{2}{*}{$\begin{array}{c}\text { No. pacientes } \\
8\end{array}$} & \multirow{2}{*}{$\begin{array}{r}\% \\
25,0\end{array}$} \\
\hline Antituberculosos & rifampicina, isoniazida, estreptomicina, pirazinamida & & \\
\hline Antibióticos y & & & \\
\hline antiparasitarios & trimetoprim, sulfametoxasol, clindamicina, dicloxacilina, primaquina & 6 & 18,7 \\
\hline Antihipertensivos & prazosín, verapamilo, captopril & 4 & 12,5 \\
\hline Otros & 5 fluorocitocina, insulina, salbutamol, prednisona & 5 & 15,6 \\
\hline \multicolumn{4}{|l|}{ Para enfermedad } \\
\hline ácido péptica & antiácidos, inhibidores $\mathrm{H}_{2}$ & 3 & 9,4 \\
\hline Antivirales & AZT, 3TC, aciclovir & 2 & 6,3 \\
\hline Complementos vitamínicos & vitamina C, sulfato ferroso, ácido fólico & 2 & 6,3 \\
\hline Anticonvulsivantes & fenitoína & 1 & 3,1 \\
\hline Antifúngico diferente a ITZ & fluconazol & 1 & 3,1 \\
\hline
\end{tabular}

diferentes tipos de micosis refleja mayor inmunosupresión y mal estado general del paciente; la enfermedad acidopéptica determina el uso de fármacos cuya interacción sobre el itraconazol ha sido reconocida en otros estudios $(3,9,16,17-18)$.

En voluntarios sanos y pacientes con micosis que reciben ketoconazol y rifampicina concomitantemente se ha reportado una disminución significativa en las concentraciones séricas de ketoconazol $(19,20)$, un imidazol con características farmacológicas similares al itraconazol. Tucker y colaboradores encontraron una interacción similar entre la rifampicina y el itraconazol en micosis sistémicas y, al igual que en el presente estudio, la reducción en las concentraciones séricas del triazol se asocia con fallas en la terapia $(3,10,13)$ y se atribuye a la inducción de la enzima citocromo P450 CYP3A4 por la rifampicina (cuadro 3).

La rifampicina se considera un potente inductor de las enzimas microsómicas intestinales y hepáticas, lo que determina, en diferentes grados, una disminución de la biodisponibilidad oral y la vida media sérica de una amplia variedad de compuestos, entre ellos el itraconazol (21). En los siete pacientes que recibieron rifampicina como parte del tratamiento antituberculoso, se documentó interacción medicamentosa, asociada con criterios de sospecha clínica o paraclínica. La disminución en los niveles séricos de itraconazol y la falta de respuesta al tratamiento se le atribuye principalmente a la administración concomitante de rifampicina y al papel de las enfermedades de base y concomitantes como favorecedoras de la interacción.

De los cinco pacientes con enfermedad acidopéptica, únicamente tres recibían tratamiento con inhibidores $\mathrm{H}_{2}$ (ranitidina) y antiácido (hidróxido de aluminio más hidróxido de magnesio). Los tres pacientes presentaron persistencia de cultivos positivos y de serología reactiva, ausencia de mejoría clínica o empeoramiento de la enfermedad. El aumento del pH gástrico por la administración de antiácido e inhibidores $\mathrm{H}_{2}$, podría explicar la disminución de la absorción del itraconazol, con la subsecuente reducción de sus niveles séricos (12,16-18).

La polifarmacia puede empeorar también la enfermedad acidopéptica o producir efectos adversos gastrointestinales, tales como vómito y diarrea, que en casos severos pueden comprometer la administración y absorción del mismo fármaco. Es importante mencionar que aunque el itraconazol es mejor tolerado que el ketoconazol (13), los efectos gastrointestinales indeseables pueden también presentarse bajo terapia con este triazol (16).

El único paciente que recibió fenitoína, no presentó ni patología de base ni concomitante pero sufría de paracoccidiodomicosis cerebral multifocal. La fenitoína es considerada como un inductor del sistema citocromo P450 microsomal $(3,22,23)$ y su acción en este paciente contribuyó, muy probablemente, a la presencia de interacción medicamentosa. 
Cuadro 3. Comparación de los principales hallazgos de los estudios de Tucker y col. vs Fernández y col.

\begin{tabular}{|c|c|c|}
\hline \multirow[b]{2}{*}{ Características } & \multicolumn{2}{|l|}{ Estudio } \\
\hline & Tucker y col. & Fernández y col. \\
\hline Número de pacientes & 10 & 21 \\
\hline \multirow[t]{4}{*}{ Tipo de micosis } & Criptococosis & Criptococosis \\
\hline & Coccidioidomicosis & Paracoccidioidomicosis \\
\hline & & Cromoblastomicosis \\
\hline & & Histoplasmosis \\
\hline Número de pacientes con niveles séricos & 10 & 18 \\
\hline \multirow[t]{4}{*}{ Enfermedades de base } & VIH-SIDA & VIH-SIDA \\
\hline & Diabetes & Diabetes \\
\hline & Trasplante & Insuficiencia renal \\
\hline & Insuficiencia renal & Ninguna \\
\hline \multirow[t]{3}{*}{ Enfermedades concomitantes } & Mycobaterias diferentes a M.tuberculosis & Tuberculosis \\
\hline & Epilepsia & Enfermedad acidopéptica \\
\hline & & $\begin{array}{l}\text { Hipertensión arterial } \\
\text { Ninguna }\end{array}$ \\
\hline \multirow[t]{3}{*}{ Fármacos involucrados en la interacción } & Rifampicina & Rifampicina \\
\hline & Fenitoína & Antiácidos \\
\hline & Carbamazepina & $\begin{array}{l}\text { Inhibidores } \mathrm{H}_{2} \\
\text { Fenitoina }\end{array}$ \\
\hline
\end{tabular}

En tres pacientes que recibieron concomitantemente itraconazol, prasozin o 5-fluorocitocina, se hallaron niveles séricos elevados de itraconazol pero presentaron criterios de falla terapéutica, la cual no se explica por el tipo de medicamentos recibidos sino, por el contrario, por el tipo y la severidad de la micosis (histoplasmosis pulmonar multifocal y cromoblastomicosis), consideradas infecciones crónicas y de difícil tratamiento $(2,3,15)$.

En conclusión, las alteraciones producidas por los fármacos administrados simultáneamente sobre la absorción (inhibidores $\mathrm{H}_{2}$, antiácidos) y metabolismo (rifampicina, fenitoína) del itraconazol, fueron los mecanismos más frecuentes de interacción farmacocinética.

En la mayoría de pacientes que presentaron sospecha clínica o paraclínica de falla terapéutica fue posible comprobar precozmente la interacción por medio de la medición de niveles plasmáticos de itraconazol por la técnica de bioensayo, por lo cual se deduce que los síntomas y los signos, especialmente clínicos, son buenos predictores de la alteración en los niveles de itraconazol.
Todos los pacientes que presentaron VIH-SIDA desarrollaron interacción medicamentosa debido a los fármacos recibidos o por trastornos gastrointestinales, como la hipoclorhidria, que disminuyen la absorción del medicamento. La tuberculosis y la enfermedad acidopéptica fueron las entidades concomitantes más frecuentes y éstas, igualmente, determinan el uso de fármacos cuya interacción sobre el itraconazol ha sido ya reconocida (rifampicina, antiácidos e inhibidores $\mathrm{H}_{2}$ ).

\section{Agradecimientos}

El grupo de investigación agradece a las doctoras Angela Restrepo, Angela Tobón, Myrtha Arango y al doctor William Mejía y a la Universidad Pontificia Bolivariana, la Corporación para Investigaciones Biológicas por hacer posible la realización de este trabajo.

\section{Referencias}

1. Souith PD. Interacciones Medicamentosas. En: Busto UE, et al. Métodos en Farmacología Clínica. Programa Regional de Medicamentos Esenciales, Programa Desarrollo de Servicios de Salud, OPS 1992: 266.

2. Múnera MI. Terapia antifúngica. En: Restrepo A, et al. Enfermedades Infecciosas. Fundamentos de Medicina. $5^{\text {la }}$. 
edición, Medellín: Corporación para Investigaciones Biológicas: 49.

3. Tucker RM, Denning DW, Hanson LH, Stevens D, Rinaldi $\mathrm{M}$, Graybill J, et al. Interaction of azoles with rifampin, phenytoin, and carbamazepine: in vitro and clinical observations. Clin Infect Dis 1992; 14: 165-74.

4. Hostetler JS. Discrepancies in bioassay and chromatography determinations explained by metabolism of itraconazole to hidroxyitraconazole studies of interpatient variations in concentrations. Antimicrob Agents Chemother 1993; 37: 2224-27.

5. BullokW.E. Histoplasma capsulatum. En : Mandell GL, et al. Principles and Practice of Infectious Disease. New York; Churchill Livingstone, 1995;2340-53.

6. Restrepo A. Paracoccidioides brasiliensis. En : Mandell, G.L, et al. Principles and Practice of Infectious Disease. New York; Churchill Livingstone, 1995; 2386-89.

7. Diamond RD. Cryptococcus neoformans. En : Mandell, G.L, et al. Principles and Practice of Infectious Disease. New York; Churchill Livingstone, 1995; 2331-40.

8. Bennett JE. Aspergillus species. En : Mandell, G.L, et al. Principles and Practice of Infectious Disease. New York; Churchill Livingstone, 1995;2386-89.

9. Piscitelli S, Flexner C, Masur H, Minor J, Polis M. Drug interactions in patients infected with human immudeficiency virus. Clin Infect Dis 1996; 23: 685-93.

10. Lake KD. Drug interactions intTransplantpPatients. Handbook of cardiac transplantation 1995: 173-200.

11. Ducharme M, Van de VeldeV, Mannens G, Slaughter R, Warbasse L, Chandrasekar P, et al. Itraconazole and hidroxyitraconazole serum concentrations are reduced more than tenfold by phenytoin (abstract). Clin Pharmacol Ther 1995; 58: 617-24.

12. Negroni R, Arechavala Al. Itraconazole: pharmacokinetics and indications. Arch Med Res 1993;24 (4):38793.
13. Lake-Bakkar G. Gastropathy and ketoconazole malabsorption in the Acquired Immunodeficiency Syndrome (AIDS). Ann Intern Med 1989; 109: 471-3.

14. Baciewicz AM, SelfT, BekemeyerW. Update on rifampin drug interaction. Arch Intern Med 1987; 147: 565-8.

15. Wagner KF. Agentes de la cromomicosis. En: Mandell G, et al. Enfermedades infecciosas. Principios y práctica. $4^{\text {ta }}$ edición. Buenos Aires: Editorial Panamericana 1997;260912.

16. Grant SM, Clissold SP. Itraconazole: a review of its pharmacodinamic and pharmacokinetic properties and therapeutic use in superficial and systemic mycoses. Drugs 1989; 37 (3): 310-44.

17. Haria M, Bryson $\mathbf{H}$, Goa K. Itraconazole. A reappraisal of its pharmacological properties and therapeutic use in the management of superficial fungal infections. Drugs, 1996;51(4): 586-620.

18. Blum RA, Hilligoss D, Garner M, Henry E, D'Andrea D, Florentino $B$, et al. Increased gastric $\mathrm{pH}$ and the bioavailability of fluconazole and ketoconazole. Ann Intern Med 1991;114:755-7.

19. Kauffman CA. Role of azoles in antifungal therapy. Clin Infect Dis 1996;22:S148-53.

20. Arredondo G, Martinez-Jorda R, Aguirre C, Suarez C. Protein binding of itraconazole and fluconazole in patient with chronic renal failure. Internat J Clin Pharmaco Ther, 1994; 32(7): 361-4.

21. Farr BM. Rifampicina. En: Mandell G, et al. Enfermedades infecciosas. Principios y práctica. $4^{\text {ta }}$ edición. Buenos Aires: Editorial Panamericana 1997;352-65.

22. Rall TW, Schleifer LS. Fármacos efectivos para el tratamiento de la epilepsia. En: Goodman A, Goodman L. Bases farmacológicas de la terapéutica. $8^{\text {va }}$ edición. México: Editorial Panamericana, 1991;440.

23. Mitchell A, Holland J. Fluconazole and phenytoin: a predictable interaction. BMJ, 1989;298:1315. 